\title{
Thin-Shell Deployable Reflectors with Collapsible Stiffeners: Experiments and Simulations
}

\author{
Lin Tze Tan* \\ University College London, London WC1E 6BT, United Kingdom \\ and \\ Sergio Pellegrino ${ }^{\dagger}$ \\ California Institute of Technology, Pasadena, California 91125 \\ DOI: $10.2514 / 1 . J 051254$
}

\begin{abstract}
This paper presents an experimental and computational study of four deployable reflectors with collapsible edge stiffeners, to verify the differences in behavior that had been predicted in a previous theoretical study. The experimental models have different geometric configurations and are made of two different plastics. Both folding experiments and vibration tests in the fully deployed configuration are carried out on each model, and it is shown that good correlation with finite element simulations can be achieved if detailed effects such as material nonlinearity, geometric imperfections, air, and gravity effects are included in the computer models.
\end{abstract}

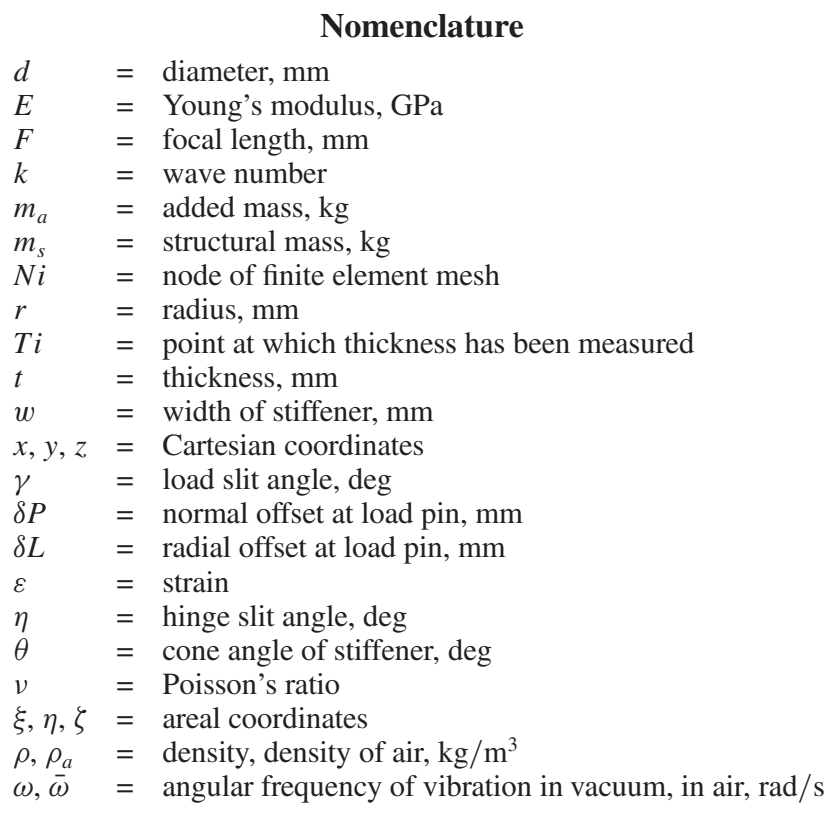

\section{Introduction}

$\mathbf{T}$ O REALIZE the full potential of high-modulus, thermally stable ultrathin materials for next generation low-cost deployable space structures will require novel structural concepts able to provide within the structure functions that were previously provided by separate structural components. The particular kind of structure that is considered in this paper, known as the springback reflector [1,2], is a thin shell deployable structure suitable for high-

Received 26 March 2011; revision received 20 June 2011; accepted for publication 10 July 2011. Copyright (C) 2011 by Lin Tze Tan and Sergio Pellegrino. Published by the American Institute of Aeronautics and Astronautics, Inc., with permission. Copies of this paper may be made for personal or internal use, on condition that the copier pay the $\$ 10.00$ per-copy fee to the Copyright Clearance Center, Inc., 222 Rosewood Drive, Danvers, MA 01923; include the code 0001-1452/12 and \$10.00 in correspondence with the CCC.

*Lecturer in Structural and Solid Mechanics, Head of Structural Engineering Group, Department of Civil Engineering, Gower Street; 1.tan@ucl.ac.uk. Member AIAA.

${ }^{\dagger}$ Joyce and Kent Kresa Professor of Aeronautics and Professor of Civil Engineering, Graduate Aerospace Laboratories, 1200 East California Boulevard, Mail Code 301-46; sergiop@ caltech.edu. Fellow AIAA. gain antennas, mirrors, etc. It is made as a single piece, for example by curing carbon-fiber prepregs on a curved mandrel. The folding scheme consists in pulling two diametrically opposite edge points of the shell towards each other, as shown in Fig. 1. The structure is launched into space in this folded configuration, held by one or more tie cables and, once in orbit, the tie cables are cut and the reflector is thus able to self-deploy.

A previous theoretical study [3] has introduced the concept of collapsible stiffeners that are an integral part of the main shell and hence can be constructed together with it. This new concept maintains the simplicity of the original springback reflector concept [2] but provides a low-cost scheme for firmly latching the structure into its deployed configuration.

For a given geometry and elastic properties of the main shell, several configurations of the stiffeners were considered in a previous paper [3] and for each configuration the geometry of the stiffeners was optimized through a sensitivity study. Thus several optimized designs were obtained, which 1) could be fully folded without permanent deformation or fracture of the material of the shell, and 2) maximized the stiffness of the shell (measured through its fundamental natural frequency of vibration) in the deployed configuration. This initial study was carried out purely numerically using two kinds of analyses, a linear eigenvalue analysis to extract the natural frequencies and eigenmodes of the reflector shell in the deployed configuration, and a large-displacement simulation of the folding of the reflector to obtain the maximum strain in the folded configuration. Both of these analyses were carried out with the ABAQUS/Standard finite element software.

The main outcome of this previous study is summarized by the force-displacement curves shown in Fig. 2. Here the unstiffened configuration, denoted by $\mathrm{O}$, has low stresses in the folded configuration but also has very low stiffness when it is deployed; the configuration with a conical edge stiffener of uniform width and with a pair of circumferential slits of equal length along the seam between the paraboloidal shell and the conical shell, denoted by $\mathrm{C} 90$, has high stiffness when it is deployed but also has very high stresses in the folded configuration; the configuration with two pairs of circumferential slits, denoted by D, has both significantly higher stiffness and an acceptable maximum stress.

The present paper presents an experimental study of four of the structural configurations considered previously [3], to verify the predicted differences in behavior. The layout of the paper is as follows. Section II describes the design and construction of four experimental models made of two different plastics. The experiments, including both folding experiments and vibration tests in the fully deployed configuration, are presented in the following section. Section IV presents the finite element models that were used to 

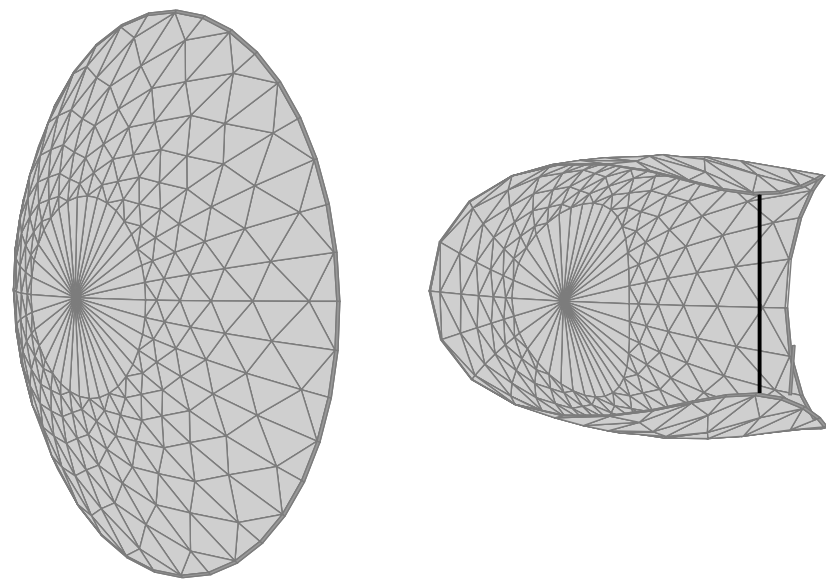

Fig. 1 Schematic illustration of springback reflector concept, deployed and folded.

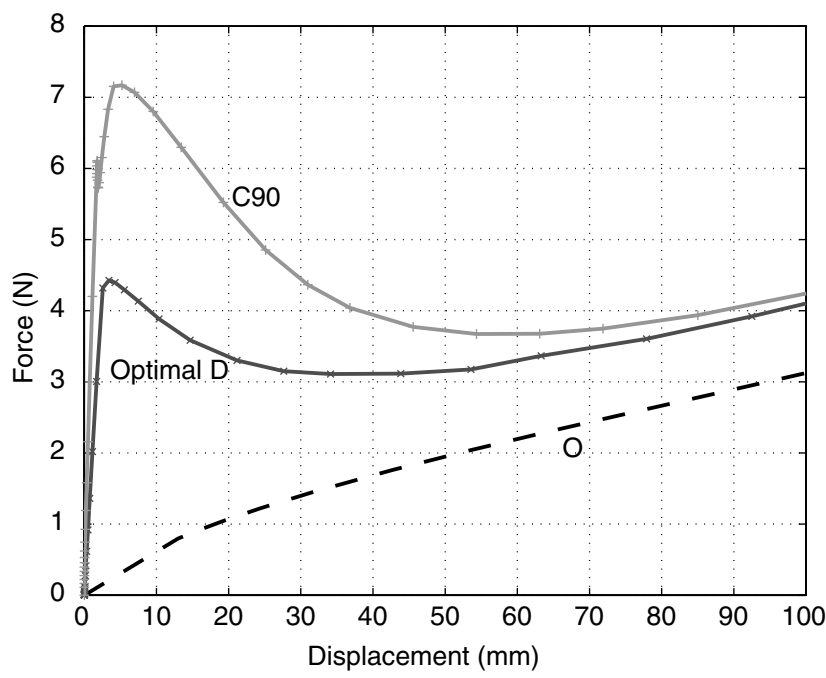

Fig. 2 Comparison of force-displacement relationships for $452 \mathrm{~mm}$ diameter reflectors that have no stiffener, $O$, or a conical edge stiffener with a single circumferential pair of slits, $\mathrm{C90}$, or two circumferential pairs of slits, D. Figure reproduced from [3]. simulate the experiments and which included four important effects: manufacturing imperfections, geometric offset of loads, self-weight, and air-structure interaction. The results from the experiments and the numerical models are presented and compared in Sec. V; a discussion follows in Sec. VI. Section VII concludes the paper.

\section{Design and Construction of Physical Models}

The same axisymmetric aperture, with equation $z=\left(x^{2}+\right.$ $\left.y^{2}\right) / 4 F$, considered in section 5 of [3] is also considered here. The focal length is $F=134 \mathrm{~mm}$ and the diameter is $d=452 \mathrm{~mm}$; the focus to diameter ratio is $F / d=0.296$. The four configurations that were studied are presented in Fig. 3 and Table 1, where each configuration is denoted with the same notation introduced in [3]. The letters $\mathrm{A}, \mathrm{C}$, and D denote configurations with a pair of cuts, a pair of slits, or two pairs of slits, respectively; where $\mathrm{A}$ and $\mathrm{C}$ are followed by a number that provides the orientation of the cuts/slits with respect to the direction in which the edge points of the reflector are pulled together for folding. A subscript denotes the cone angle of the stiffener.

The first configuration, Fig. 3a, is unstiffened. The second and third configurations, Figs. $3 \mathrm{~b}$ and $3 \mathrm{c}$, have a cone angle of $50 \mathrm{deg}$. The second configuration, denoted by $\mathrm{A} 90-\mathrm{CO}_{50}$, has a $20 \mathrm{~mm}$ wide edge stiffener with a pair of diametrically opposite cuts in the stiffener and also a pair of diametrically opposite slits, each subtending an angle of $4 \mathrm{deg}$. The third configuration, denoted by $\mathrm{D}_{50}$, has a $20 \mathrm{~mm}$ wide stiffener with two pairs of diametrically opposite slits, one subtending $8 \mathrm{deg}$ and one $24 \mathrm{deg}$, and no cuts. The last configuration, denoted by $\mathrm{D}_{90}$, has a $20 \mathrm{~mm}$ wide edge stiffener that lies in a plane; it has two pairs of slits matching those in the third configuration.

All models were vacuum formed from $1 \mathrm{~mm}$ thick sheets of a thermoplastic polymer (see below for details) on a parabolic mold with a conical edge, made from Renshape 460 polyurethane board. Vacuum forming is an inexpensive and seamless construction method capable of achieving the high precision needed to ensure that the radius of curvature at the transition between the dish and the stiffener is both uniform and quite small. The sheet material is held above the mold and heated to about $200^{\circ} \mathrm{C}$; vacuum is created between the mold and the sheet, through small holes in the mold, to pull the sheet onto the mold; and then the material is allowed to cool slowly to avoid high residual stresses.

The skirt was trimmed to the correct width using a band saw and the edges were sanded. Cuts and slits were created by running a scalpel along the center line. Typically, the geometric accuracy of the formed shell is a very close approximation to the shape of the mold,

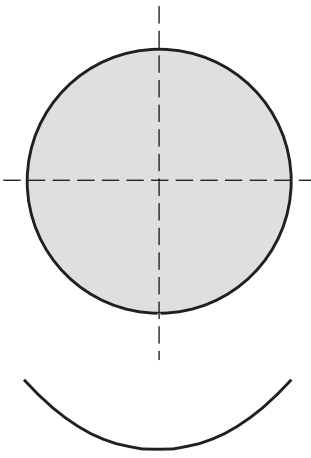

a) Model 0

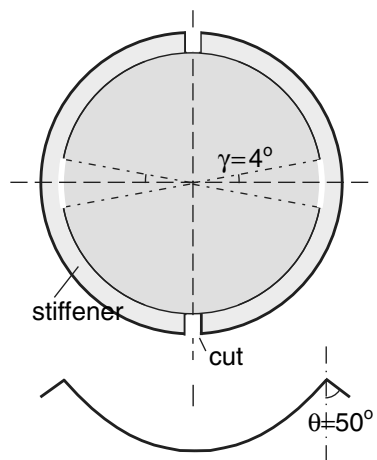

b) Model A90-C0 50

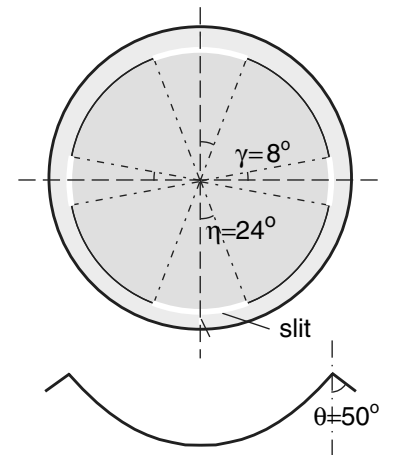

c) Model $\mathbf{D}_{\mathbf{5 0}}$

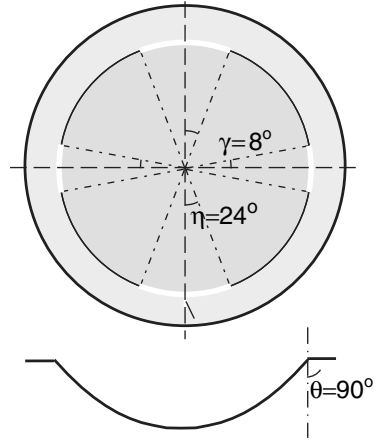

d) Model $\mathbf{D}_{90}$

Fig. 3 Four configurations studied in this paper; the direction of folding is vertical.

Table 1 Geometric properties of models tested

\begin{tabular}{|c|c|c|c|c|c|c|c|}
\hline Model & Material & $w, \mathrm{~mm}$ & $\theta, \operatorname{deg}$ & \# cuts & \# slits & $\eta$, deg & $\gamma, \operatorname{deg}$ \\
\hline $\mathrm{O}$ & PETG & - & - & - & - & {[} & - \\
\hline $\mathrm{A} 90-\mathrm{CO}_{50}$ & PETG & 20 & 50 & 2 & 2 & 4 & - \\
\hline$D_{50}$ & Polycarbonate & 10 & 50 & 0 & 4 & 24 & 8 \\
\hline$D_{90}$ & PETG & 20 & 90 & 0 & 4 & 24 & 8 \\
\hline
\end{tabular}


Table 2 Properties of polycarbonate and PETG, from supplier data

\begin{tabular}{lcc}
\hline \hline Property & Polycarbonate & PETG \\
\hline Density, $\mathrm{kg} / \mathrm{m}^{3}$ & 1,200 & 1,270 \\
Young's modulus, $\mathrm{GPa}$ & 2.30 & 2.05 \\
Tensile strength, $\mathrm{MPa}$ & 70 & 50 \\
Flexural strength, $\mathrm{MPa}$ & 90 & 80 \\
Poisson's ratio & 0.4 & 0.3 \\
Elongation at yield, $\%$ & 7 & 5 \\
Glass transition temp, ${ }^{\circ} \mathrm{C}$ & 148 & 81 \\
\hline \hline
\end{tabular}

however, the thickness of the shell is usually nonuniform because the sheet stretches by different amounts depending on the slope of the mold (see Sec. IV.A for details).

The two thermoplastic polymers that were used were polycarbonate and polyethylene terephthalate glycol modified (PETG, which has the trade name Vivak). Polycarbonate has higher flexural and tensile strengths and can undergo higher strains, while having a lower density. PETG on the other hand is easier to vacuum form and is less sensitive to moisture. A full set of material properties for these two materials is provided in Table 2.

Tension tests were carried out on $200 \mathrm{~mm}$ long dogbone specimens with a $90 \mathrm{~mm}$ long central region with uniform width of $18 \mathrm{~mm}$, at a rate of $0.05 \mathrm{~mm} / \mathrm{s}$. Strain gauge rosettes were glued onto the midsection of each specimen. Each specimen was loaded cyclicly to $30 \%$ of the respective yield strain, i.e., $1.6 \%$ for PETG and $2.2 \%$ for polycarbonate; these strain values are equivalent to about $60 \%$ of the respective tensile strengths.

The test results for polycarbonate, Fig. 4a, show that its behavior is nonlinear (softening), with a tangent modulus that decreases from

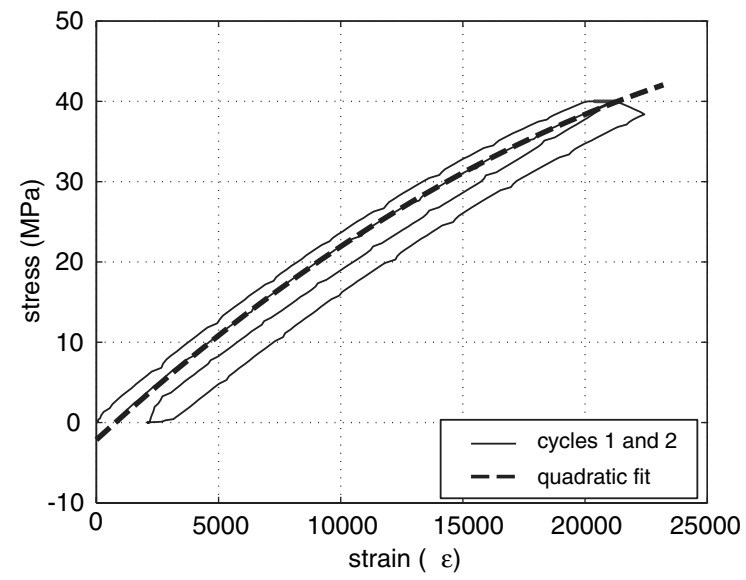

a) Polycarbonate

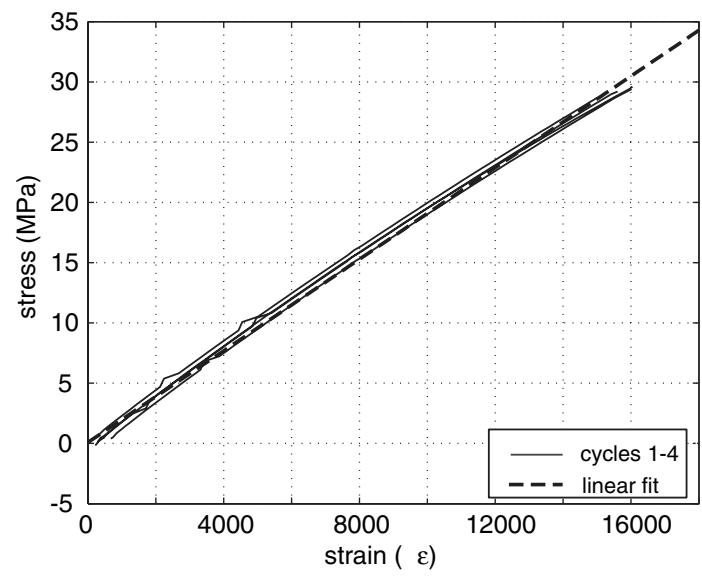

b) PETG

Fig. 4 Uniaxial behavior of polycarbonate and PETG.
Table 3 Measured and quoted properties of PETG and polycarbonate

\begin{tabular}{lcccc}
\hline \hline & \multicolumn{2}{c}{ Measured values } & \multicolumn{2}{c}{ Supplier's data } \\
\cline { 2 - 5 } Material & $E, \mathrm{GPa}$ & $v$ & $E, \mathrm{GPa}$ & $v$ \\
\hline PETG & 1.86 & 0.3648 & 2.05 & 0.3 \\
Polycarbonate & $2.70-1.38$ & 0.3328 & 2.3 & 0.4 \\
\hline \hline
\end{tabular}

2.70 to $1.38 \mathrm{GPa}$. Its nonlinearity corresponds to a $38 \%$ decrease in stiffness when the specimen is subjected to a strain of $2 \%$; this variation is included in the finite element model presented in Sec. IV. The measured value for Poisson's ratio is 0.333 , hence the differences between the supplier's data and the experimentally measured values are $3-40 \%$ for the modulus and $17 \%$ for the Poisson's ratio.

The stress-strain graph for Polycarbonate shows a quadratic trend (denoted by the dotted line in Fig. 4a), while a loading and unloading cycle shows that this material has a tendency to develop a permanent set.

The test results for PETG, Fig. 4b, are much closer to linear and there is very little permanent set. The measured values for the modulus and Poisson's ratio are $1.86 \mathrm{GPa}$ and 0.365 , representing differences of $9 \%$ from the modulus and $22 \%$ from the Poisson's ratio provided by the supplier. A comparison of measured vs supplierprovided material properties is given in Table 3 .

\section{Experiments}

Two types of experiments were carried out on each model: 1) folding tests, to characterize both the initial stiffness of the structure and the force-displacement relationship during folding; and 2) vibration tests, to measure the natural frequencies of the fully deployed structure.

\section{A. Folding Tests}

All tests were carried out with an Instron 5564 materials testing machine operating in displacement controlled mode to capture any instabilities. The test fixtures were specially designed to provide $2 \mathrm{~mm}$ diameter socket joints connecting to $2 \mathrm{~mm}$ diameter steel pins attached to the edge of each model, as shown in Fig. 5a. Friction in this joint introduces only a very small disturbance, due to the small diameter of the pin head. The test fixtures were designed such as to allow large rotations of the attachment points of the model. Also, the fixtures were mounted next to one side of the testing machine, to maximize the space available for the model to deform when it starts folding; note that the load cell was attached to the bottom plate of the testing machine instead of the moving cross head. A detailed view of the attachment between fixture and structure is shown in Fig. 5b; more details are provided in Fig. 6.

The vertical force applied by the bottom fixture, measured by a $3 \mathrm{kN}$ capacity load cell, and the displacement of the cross head of the Instron testing machine were recorded at a rate of $0.5 \mathrm{~s}$.

Each test consisted in moving the cross head of the testing machine to a position such that the distance between the socket joints approximately matched the distance between the pin heads on the undeformed model. Then the pin heads were pushed into the socket joints and the cross head position was again adjusted until the force measurement by the load cell became equal to half the weight of the model. The force measurement was then set to zero and the loaddisplacement relationship was measured over a displacement range of up to $260 \mathrm{~mm}$, corresponding to approximately a $50 \%$ reduction of the distance between the two pin heads. Models A90- $\mathrm{C}_{50}$ and $\mathrm{D}_{50}$ appeared to be more prone to permanent deformation than the other models and hence the tests on these models were stopped at around $60 \%$ of the full displacement.

Since all models were held vertical during the tests, determining the initial configuration was problematic for some models. Part of the problem is that the initial configuration is not fully unstressed, because of the model's self-weight and the associated reactions at the pins. The effects of changing the initial test configuration for each 


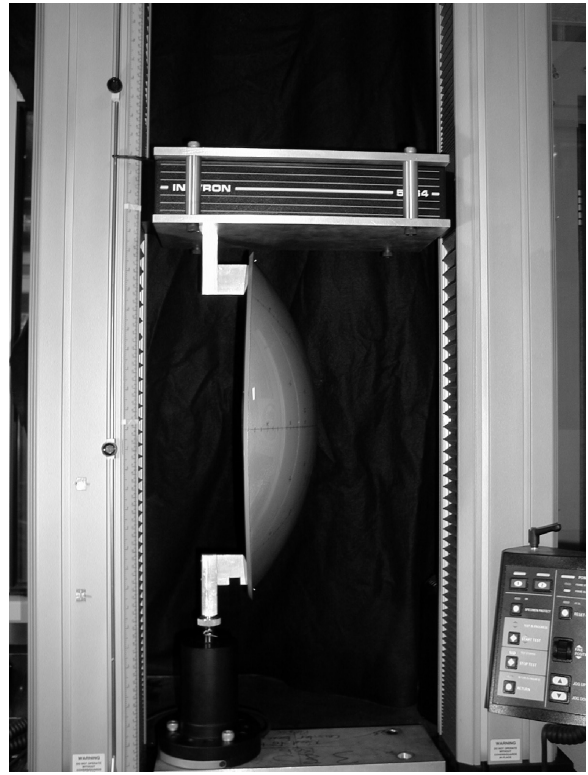

a) Arrangement of model in testing machine

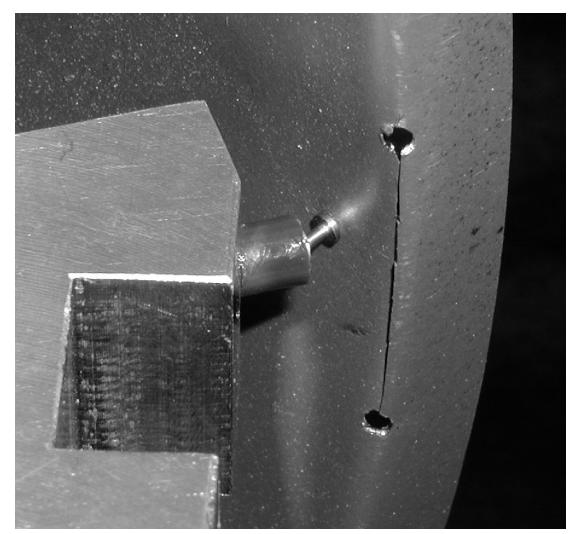

b) Closeup of attachment

Fig. 5 Experimental setup for folding test.

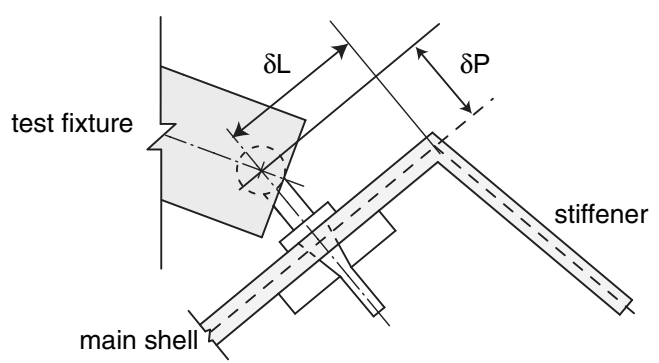

a) Load and pin offsets

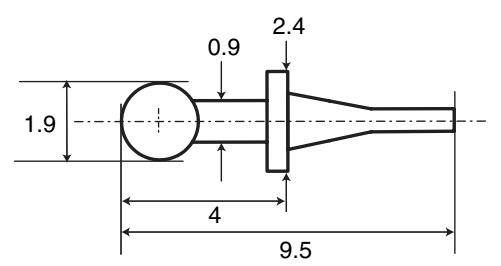

b) Pin dimensions in $\mathbf{m m}$

Fig. 6 Offsets introduced by size and location of pin.

model were examined by carrying out two or three separate tests with different initial conditions. The imposed displacement rate was also found to affect the results. After some preliminary tests had shown that rates faster than $1 \mathrm{~mm} / \mathrm{s}$ had the effect of changing the measured response, a standard displacement rate of $1 \mathrm{~mm} / \mathrm{s}$ was chosen for the folding tests.

\section{B. Vibration Tests}

Modal identification tests were carried out by applying a random dynamic excitation to each model and by measuring the response of the model with a Polytec PSV300 scanning laser vibrometer [4]. The excitation of the model was achieved via a PCB piezotronics [5] model 086D80 impulse hammer fitted with a piezoelectric sensor head. The hammer impulse test imparts a nearly constant force over a broad range of frequencies, and hence excites many resonances in each test. The hammer was mounted at a fixed position near the rim of the dish and hence provided an excitation to the same point of the model for every measurement. The dynamic responses of nine target points on each model (forming a circular grid of eight points plus one point at the center) were measured. For greater accuracy the impulse and the corresponding displacements and velocities of each target point were averaged over three separate excitations; once the measurements at a target point had been completed the laser beam was automatically moved to the next target point.

Besides measuring the eigenfrequencies of a structure, the laser vibrometer is also capable of measuring and computing the corresponding eigenmodes, thus the different natural frequencies can be visually correlated with the respective modes. Although the mode of greatest interest is the softest, i.e., the lowest bending mode, the first six modes of each model were measured.

Each measurement was repeated 3 times and the results were averaged. Once the average response of all target points had been obtained, the fast Fourier transform (FFT) of the signals was computed to obtain the frequency response functions and the mode shapes.

Two different boundary conditions were used:

1) Clamped: Model mounted vertically, bolted through the center to a vertical arm and attached to a massive steel block, Fig. 7a.

2) Free-free: Model mounted horizontally and facing either "cupup" or "cup-down," suspended from three vertical cords from a rigid ring of similar diameter, Fig. 7b. The lengths of the suspension cords were such that the rigid-body vertical mode had a frequency of $1 \mathrm{~Hz}$, to separate the rigid body modes from the flexural modes (which have frequencies of around $10 \mathrm{~Hz}$ ). Horizontal elastic ties were used to prevent horizontal rigid body motions.

The coherence values, i.e., the ratios of the frequency response functions [6], are often used to establish the accuracy and validity of vibration measurements. Values close to 1 are most desirable. Coherence values greater than 0.8 were obtained for all configurations apart from Model $\mathrm{O}$. This axisymmetric model has an infinite number of coincident modes and hence it is impossible to obtain a good coherence by applying a single excitation.

\section{Finite Element Models}

This section describes a series of experimental details that need to be captured by the numerical models to obtain sufficiently representative simulations of the tests presented in the previous section. 


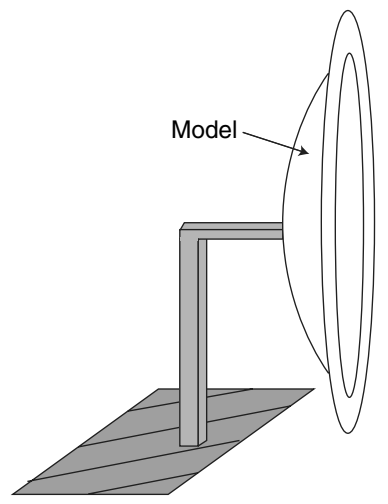

a) Clamped

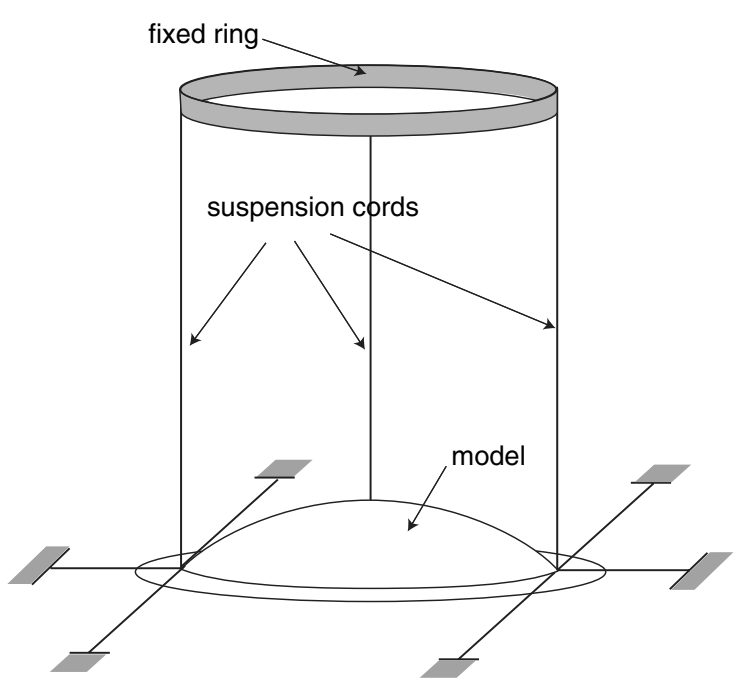

b) Free-Free

Fig. 7 Vibration test setups.

The effects that need to be captured include key geometric imperfections in the models, air and gravity effects. Details on how these effects were modeled are provided in this section.

For a symmetric dish only a quarter of the whole structure was analyzed, using the appropriate boundary conditions. Rigid body motions were constrained by fixing the center of the reflector. Consistent convergence was achieved with a fine mesh of general purpose, linear three-noded triangular shell elements (S3R), average density of 1000 elements per quarter mesh, together with the default ABAQUS iteration control parameters. The slits between the dish and the stiffener were modeled by defining two separate sets of geometrically coincident nodes on either side of each slit. The cuts in the stiffener were modeled by simply leaving the respective edges of the stiffener free from any symmetry boundary conditions.

All simulations were performed with ABAQUS/Standard [7] running on a Pentium III $1 \mathrm{GHz} \mathrm{PC}$; the average run time was less than $20 \mathrm{~min}$. A typical analysis consisted of two steps, the first being a linear eigenvalue analysis (using the *FREQUENCY,SUBSPACE options) to extract the natural frequencies and eigenmodes of the reflector in its deployed configuration, and the second step being a geometrically nonlinear simulation of the folding of the reflector. Moving boundary conditions were applied to the rim of the reflector, to impose a total displacement of $d / 3$ and the *STEP, NLGEOM, and *STATIC options were used to simulate this folding regime.

Regarding the material behavior, it was shown in Sec. II that while PETG is linear for stresses up to $30 \mathrm{MPa}$, polycarbonate has an approximately quadratic stress-strain relationship. Hence for the $\mathrm{D}_{50}$ model a nonlinear elastic isotropic material behavior was defined, using a user-defined subroutine (UMAT in ABAQUS/Standard [7]) to provide a variable modulus as a function of the current strain. The modulus values were obtained by fitting a quadratic polynomial through the results in Fig. 4, and by differentiating this analytical relationship. The resulting expression for the modulus was:

$$
E_{\mathrm{pc}}=2.70-60 \epsilon \quad(\mathrm{GPa})
$$

Four other important issues that had to be captured in the finite element models are discussed next.

\section{A. Geometric Imperfections}

The vacuum-forming process stretches the $1 \mathrm{~mm}$ thick sheet material by variable amounts, which depend on the ratio between the surface area and its projection onto the $x-y$ plane. One would expect the deformation of the material to be axisymmetric, like the mold shape, and hence the thickness of all models should be $1 \mathrm{~mm}$ at the center, decreasing in the meridional direction.
Figure $8 \mathrm{a}$ is a graph of the thickness of model $\mathrm{D}_{50}$, measured at 28 points on 8 meridians. Note that the last two points on each curve lie on the stiffener. The largest difference along one meridian is $0.43 \mathrm{~mm}$, i.e., a $47 \%$ difference between the center and the rim. Although the thickness variation trends follow the expected pattern, there is also considerable asymmetry. The largest discrepancies on any given circumference occur near the rim, with a maximum variation of $0.23 \mathrm{~mm}$ in the stiffener. The thickness distributions are different in the four models and, whereas the model D50 had a thickness distribution with an axis of symmetry, the other models had no discernible symmetry patterns.

Considering that the flexural rigidity of a thin shell is proportional to the cube of the thickness, the measured thickness variations are significant and need to be included in the finite element models.

The mapping of the thickness point data to the finite element mesh was accomplished through the use of areal coordinates [8]. Consider a triangle with corners at three known points $\mathrm{T} 1=\left(x_{1}, y_{1}\right)$, $\mathrm{T} 2=\left(x_{2}, y_{2}\right)$, and $\mathrm{T} 3=\left(x_{3}, y_{3}\right)$ at which the thicknesses $t_{1}, t_{2}, t_{3}$, respectively, have been measured. First we define an areal coordinate system, $\xi, \eta, \zeta$ where $\mathrm{T} 1$ has areal coordinates $(1,0,0)$, and likewise $\mathrm{T} 2$ and $\mathrm{T} 3$ have coordinates $(0,1,0)$ and $(0,0,1)$.

Assume that node $N i=(x, y)$ of the predefined finite element mesh lies within triangle T1T2T3. If we then divide T1T2T3 into three triangles sharing the vertex $N i$, the areas of these triangles are proportional to the areal coordinates of $N i$ : the actual areal coordinates are obtained by dividing the areas of the three triangles by the area of T1T2T3. These areas are calculated using the formula [9]

$$
\begin{gathered}
\xi=\frac{\left(y_{2}-y_{3}\right)\left(x-x_{3}\right)+\left(x_{3}-x_{2}\right)\left(y-y_{3}\right)}{\left(y_{2}-y_{3}\right)\left(x_{1}-x_{3}\right)+\left(x_{3}-x_{2}\right)\left(y_{1}-y_{3}\right)} \\
\eta=\frac{\left(y_{3}-y_{1}\right)\left(x-x_{3}\right)+\left(x_{1}-x_{3}\right)\left(y-y_{3}\right)}{\left(y_{3}-y_{1}\right)\left(x_{2}-x_{3}\right)+\left(x_{1}-x_{3}\right)\left(y_{2}-y_{3}\right)} \\
\zeta=1-\xi-\eta
\end{gathered}
$$

as this only requires the cartesian coordinates of the vertices which are readily available. A value between 1 and 0 of all three areal coordinates indicates that $N i$ lies within T1T2T3.

A Matlab script was written to calculate the areal coordinates of every node in the mesh to check if the node lies within the triangle. If any of the three areal coordinates is either negative or larger than 1 the program concludes that $\mathrm{N} 1$ does not lie within the current triangle. It then moves on to the next triangle and continues searching until it has found the triangle in which $\mathrm{N} 1$ lies. Once this has been established, it assigns a thickness $t_{N i}$ to $N i$ by interpolation between the measured 


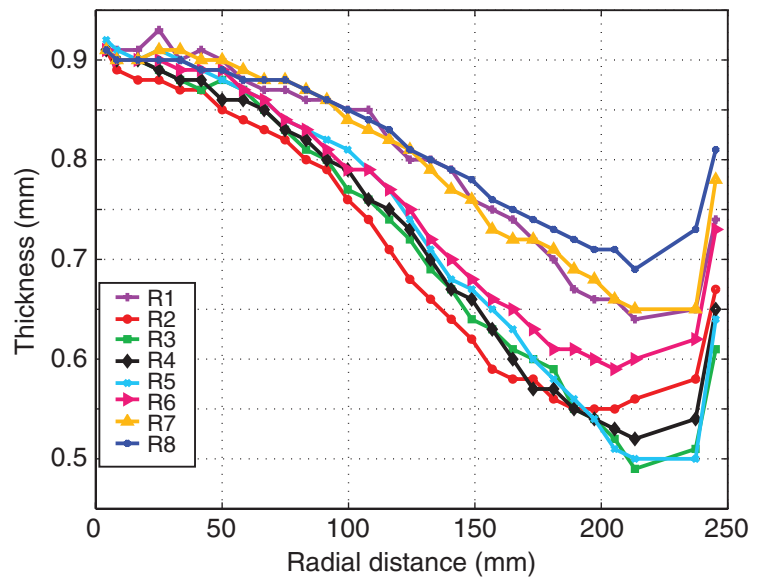

a) Meridional variation

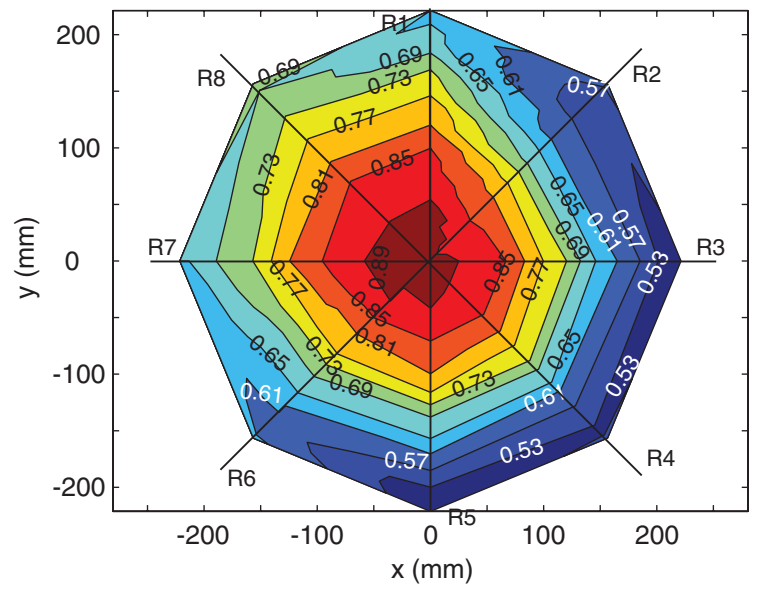

b) Contour plot

Fig. 8 Thickness measurements (in $\mathbf{m m}$ ) for model $\mathbf{D}_{50}$

thicknesses at the three vertices of the triangle containing $N i$, using the areal coordinates of the node in question, i.e.

$$
t_{N i}=t_{1} \xi+t_{2} \eta+t_{3} \xi
$$

The thicknesses at all of the nodes were found and then fed into the ABAQUS model by means of the "*Nodal Thickness" command, wherein the thickness of each node has to be specified.

\section{B. Load and Pin Offsets}

Other significant details are the exact locations of the points where the (equal and opposite) external forces are applied to the model and

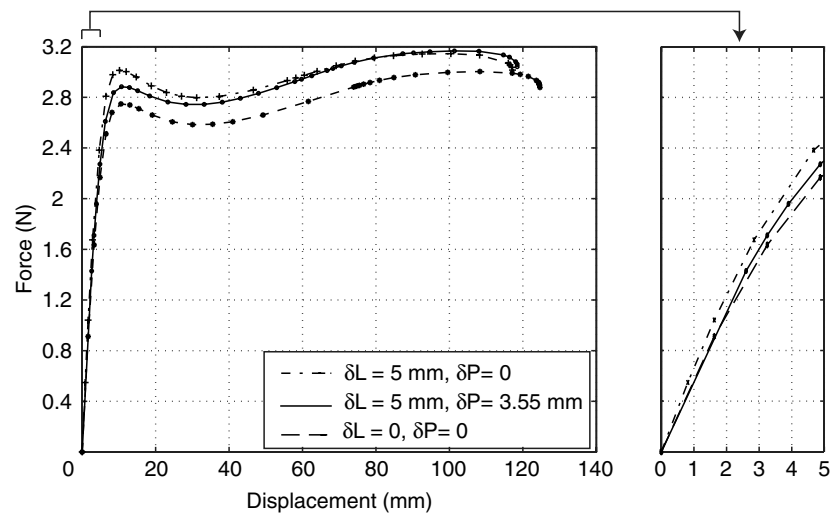

Fig. 9 Finite element analysis results showing effects of load and pin offsets. the distance between the actual point of application of these forces and the midsurface of the model. In the models that have slits in the rim, the load pins had to be moved radially inward by an amount $\delta L=5 \mathrm{~mm}$, which has the effect of changing the deployed stiffness of the model. Details of the connection and the dimensions of the pin are shown in Fig. 6. Also, in all models these externals loads are applied at a small distance $\delta P=3.55 \mathrm{~mm}$ away from the midsurface of the shell, due to the length of the load pins. Both of these offsets were introduced in the ABAQUS model, by attaching to the parabolic shell a rigid beam element of length $\delta P$, at a point that is at a distance $\delta L$ from the edge of the shell.

The effects of these offsets were investigated one by one, by running a full folding simulation for each of the following three cases: 1) shell loaded at the rim $(\delta L=0)$ with loading pin of zero length $(\delta P=0)$, which was the case considered in the finite element simulations presented in section 5.A of [3];2) shell loaded at an offset $\delta L=5 \mathrm{~mm}$ with loading pin of zero length $(\delta P=0)$; and 3) shell loaded at an offset $\delta L=5 \mathrm{~mm}$ through loading pin with $\delta P=3.55 \mathrm{~mm}$, the experimental configuration.

The results of these analyses for model $D_{50}$ are shown in Fig. 9. Both the snapping force and the initial stiffness in the deployed configuration with both load and pin offsets, i.e., case 3 , are about $5 \%$ higher than the configuration loaded at the rim through a loading pin of zero length, case 1 . However, it is interesting to note that if one considers only the load offset, case 2 , then the stiffness increases by $34 \%$ and the snapping force by about $10 \%$. Hence, the further addition of the pin offset reduces the stiffness by $22 \%$ and the snapping force by $4 \%$.

\section{Self-Weight}

The vibration tests were performed with the models held either vertical or horizontal. In a weightless environment there would be, of course, no difference; under gravity the bending modes of the structure are not significantly affected, but gravity has some effect on modes that involve significant rigid body motion. Table 4 presents the first six natural frequencies, obtained from ABAQUS/Standard, for a model that is held clamped either vertical or horizontal, and with or without gravity. The majority of the modes are practically unaffected by gravity. Mode six is the only mode that is significantly affected by the inclusion of gravity; this is because it is nearly a rigid body translation in the vertical direction.

The gravity environment was simulated in ABAQUS as a distributed load (*DLOAD option) of given acceleration magnitude and direction. Gravity was applied in the positive $x$ and $z$ directions, and the negative $z$ direction to simulate the vertical, horizontal cupup, and cup-down configurations (note that the coordinate system has been defined in Sec. II). A geometrically nonlinear static analysis step with the gravity load applied was carried out first, and this was followed by a vibration frequency analysis. The configuration with the suspension cords was not analyzed.

Figure 10 shows the mode shapes for model $\mathrm{D}_{90}$. Modes $1-3$ are rotational modes. It is worth noting that this configuration with four slits and a horizontal stiffener has two closely spaced bending modes, modes four and five, whereas for the three other configurations tested mode four is the only bending mode below $20 \mathrm{~Hz}$. These bending modes became the main focus of the study.

Table 4 Natural frequencies (in $\mathrm{Hz}$ ) of clamped model $\mathrm{D}_{\mathbf{9 0}}$

\begin{tabular}{ccccc}
\hline \hline & No gravity & \multicolumn{3}{c}{ Gravity } \\
\cline { 3 - 5 } Mode No. & & $\begin{array}{c}\text { Horizontal } \\
\text { (cup-up) }\end{array}$ & $\begin{array}{l}\text { Horizontal } \\
\text { (cup-down) }\end{array}$ & Vertical \\
\hline 1 & 2.0 & 1.7 & 2.1 & 1.9 \\
2 & 2.0 & 1.7 & 2.2 & 2.0 \\
3 & 2.1 & 2.1 & 2.2 & 2.2 \\
4 & 16.6 & 16.8 & 16.4 & 16.6 \\
5 & 18.7 & 18.8 & 18.5 & 18.6 \\
6 & 41.4 & 37.3 & 44.3 & 38.5 \\
\hline \hline
\end{tabular}




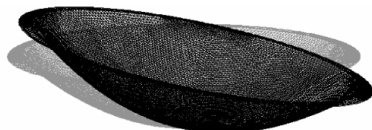

a) Mode 1

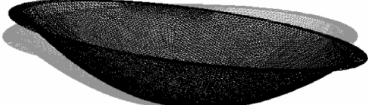

c) Mode 3

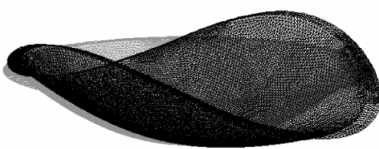

e) Mode 5

Fig. 10 Mode shapes of model $D_{90}$ clamped at the center.

\section{Air-Structure Interaction}

When a vibrating structure is in contact with a fluid medium it will invoke vibrations in the fluid. The vibration response changes at the coincidence frequency, at which point the fluid loading effect on the structure changes providing a nonstructural mass to providing radiation damping [10]. If the structure vibrates at a lower frequency than this transition, it accelerates a thin layer of fluid whose thickness decreases as the frequency increases. Hence the fluid behaves as an added mass that reduces the natural frequency of the structure.

To account for this interaction, one needs to estimate the added mass of the air. Southwell [11] and Lamb [12] considered vibrations in a plate surrounded by a fluid, but the vibrations considered were rigid body motions and hence not applicable to the particular mode that is of greatest interest in the present study. Kukathasan and Pellegrino [13] presented a method of estimating this added mass for a membrane structure, and this procedure can be adapted to plate structures or shallow shells as follows.

The circular frequency $\omega$ of a harmonic flexural wave in a plate of modulus $E$, Poisson's ratio $v$, density $\rho$, and thickness $t$, in vacuum, is given by [14]:

$$
\omega=k^{2} t \sqrt{\frac{E}{3 \rho\left(1-v^{2}\right)}}
$$

Here $k=2 \pi / \lambda$ is the wavenumber, i.e., the number of wavelengths $\lambda$ over $2 \pi$. Solving for $k$ :

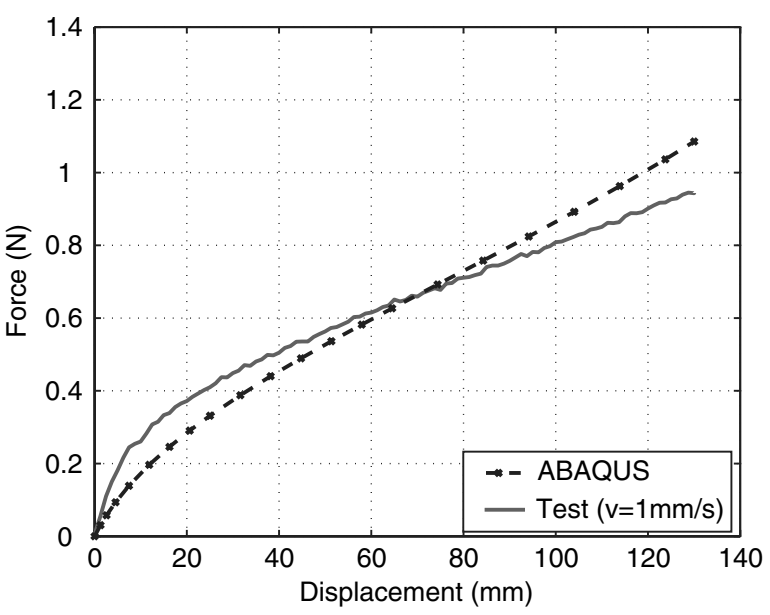

Fig. 11 Folding behavior of model $\mathrm{O}$.

$$
k=\sqrt{\frac{\omega}{t} \sqrt{\frac{3 \rho\left(1-v^{2}\right)}{E}}}
$$

Assuming that the flexural wave remains unchanged if the plate is in air of density $\rho_{a}$, the thickness of air that participates in the vibration is approximately $\rho_{a} / k$ on each side of the plate. Hence the total added mass $m_{a}$ in the case of a circular disk of radius $r$ can be simply estimated as

$$
m_{a}=2 \frac{\rho_{a}}{k} \pi r^{2}
$$

and this added mass lowers the vibration frequency of the plate to

$$
\bar{\omega}=\omega \sqrt{\frac{m_{s}}{m_{s}+m_{a}}}
$$

where $m_{s}$ is the total mass of the plate.

A more refined estimate could be obtained by introducing in the model a finite-sized air box surrounding the shell structure; the details are presented in [13]. In the present study Eqs. (7-9) have been used to determine the added-air correction for the in vacuo vibration frequencies of each experimental model, initially estimated with ABAQUS.

\section{Results}

\section{A. Folding Tests}

The measured force-displacement relationships for models $\mathrm{O}$, A90-C $0_{50}, \mathrm{D}_{50}$, and $\mathrm{D}_{90}$ are plotted in Figs. 12-14 together with the results of the corresponding ABAQUS simulations of the folding process. Note that in each case the displacement plotted is half the measured cross head displacement, i.e., the displacement of one edge if the other edge is also moved by an equal amount.

The effects of changing the initial configuration for a test on a particular model can be seen in Figs. 11-14. The effect is most noticeable in Fig. 13 where changing by $4 \mathrm{~mm}$ the distance between the pin heads and setting to zero the force on the model in this new initial configuration led to a $40 \%$ difference in the force value at the knee of the curve.

Figure 14 examines the effect of changing the speed of the cross head; note that there is a decrease of nearly $20 \%$ in the force plateau when the speed of the cross head is doubled. To avoid this force reduction all other tests were carried out at the lower speed of $1 \mathrm{~mm} / \mathrm{s}$.

\section{B. Vibration Tests}

Table 5 presents a comparison between the measured natural frequencies of the four models and the ABAQUS predictions,

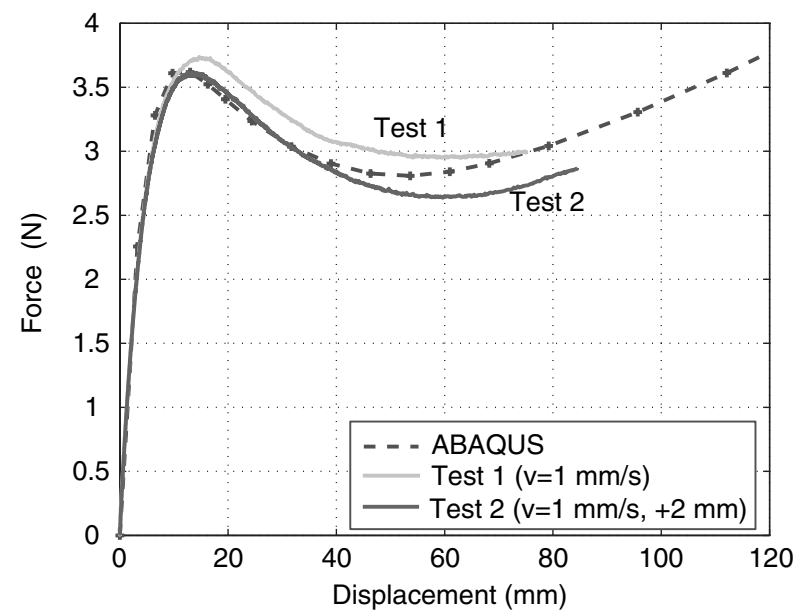

Fig. 12 Folding behavior of model $\mathrm{A} 90-\mathrm{C0}_{50}$. Tests were carried out with different starting configurations. 


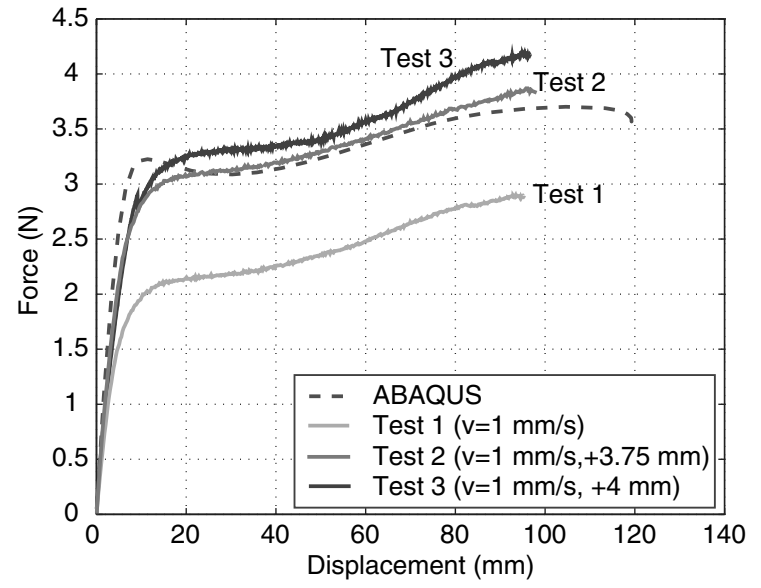

Fig. 13 Folding behavior of model $D_{50}$. Tests were carried out with different initial configurations.

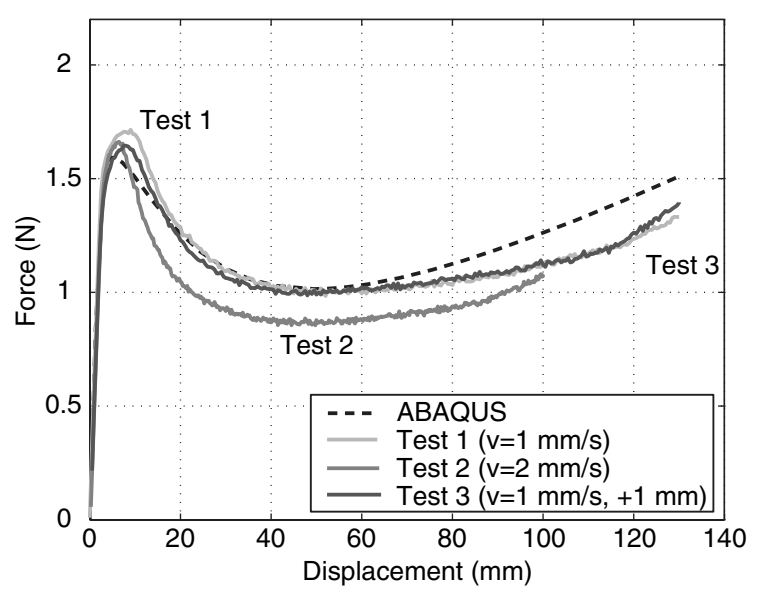

Fig. 14 Folding behavior of model $D_{90}$. Tests were carried out at different speeds.

without air effects. The measured thickness distributions have been included in the ABAQUS models. The effects of the added air mass have been estimated using Eq. (9) and the corrected frequencies are presented in the penultimate column of the table. It can be seen that once air effects have been accounted for, the error is typically in the $2-3 \%$ range, with a maximum of $5.6 \%$. These small discrepancies are most likely due to the approximations made in the evaluation of the added mass effect, e.g., the dish was approximated as a flat disc.

\section{Discussion}

Regarding the folding experiments and simulations, there is rather poor correlation between the measured response and the ABAQUS simulation for the unstiffened design (model O), with discrepancies of up to $16 \%$. This demonstrates the difficulty of accounting for the effects of gravity on a very flexible, nonlinear structure. Model $\mathrm{O}$ is the only one without an edge stiffener to maintain the surface in the correct shape and hence is the most sensitive to gravity loading. The remaining models generally show very good correlation with the test results.

The two models with the highest stiffness, model A90- $\mathrm{CO}_{50}$ and model $\mathrm{D}_{90}$, show the best correlation between experiment and simulation. For model A90-C0 ${ }_{50}$, Fig. 12, the best correlation is in the case of test 2. However after a displacement of about $40 \mathrm{~mm}$ the discrepancy increases and reaches a maximum deviation of $8 \%$, though the general trend is similar. Although the initial stiffness for test 1 , with a starting configuration that is $2 \mathrm{~mm}$ shorter, is marginally nearer to the ABAQUS model, in this test the snap is at a higher force and the response deviates even further in the postbuckling range.

Figure 14 shows the force-displacement relationship for model $\mathrm{D}_{90}$ (recall that this is the near optimum configuration obtained in [3]). Although the correlation between experimental and computational results is not as good as for model $\mathrm{A} 90-\mathrm{CO}_{50}$, the initial stiffness of this model has still been captured very accurately. The physical model snaps at a force that is $6 \%$ higher. Neglecting test 2 , which was conducted at a faster rate, the results from tests 1 and 3 are almost identical, indicating that a $1 \mathrm{~mm}$ change in the starting configuration has little effect. The measurements in the postbuckling range agree very well with the ABAQUS simulation, up to a displacement of $60 \mathrm{~mm}$; beyond this value, the results deviate by up to a maximum of $\approx 13 \%$. These lower postbuckling forces can be attributed to imperfections in the model.

Model $\mathrm{D}_{50}$ is the only one of the four dishes which was manufactured from Polycarbonate. The correlation between experimental and computational results, see Fig. 13 is the least good among all stiffened dishes. The experimental curves tend to have a lower initial stiffness and a less pronounced snap occurring at lower forces. Although the nonlinear elastic behavior of polycarbonate was accounted for in the simulation, its hysteretic behavior and the permanent set observed in the material tests were not included in the model. These effects may account for the discrepancies that have been observed.

Other factors that may have affected all of the models are geometric differences between computational and physical models such as rounding off and extra thickness at the rim of the dish or inaccuracies in the slits; the slits are difficult to manufacture accurately and even small imperfections are likely to have a significant influence on the behavior of the structure near the snap.

\section{Conclusions}

The initial aim of this experimental study of deployable reflectors with collapsible edge stiffeners was to verify the computational approaches that had formed the basis for the study presented in the theoretical part of this research, using foldable small scale models with a range of different geometric configurations. During the course of the study it was realized that to achieve a good degree of correlation it is necessary to include in the computer models detailed effects such material nonlinearity, geometric imperfections, air, and gravity effects. Once these effects had been captured then both the folding simulation results and the linear vibration behavior were found to be very accurate.

Table 5 Frequency comparison (all values in $\mathrm{Hz}$ )

\begin{tabular}{lccccc}
\hline \hline Model & Bending mode & Measured & ABAQUS & Air mass correction & Error \\
\hline $\mathrm{O}$ & 1 & 5.00 & 5.80 & 4.78 & $4.44 \%$ \\
$\mathrm{~A} 90-\mathrm{C0}_{50}$ & 1 & 14.89 & 17.00 & 15.05 & $1.07 \%$ \\
$\mathrm{D}_{50}$ & 1 & 13.44 & 14.71 & 12.91 & $3.94 \%$ \\
$\mathrm{D}_{90}$ vertical & 1 & 14.69 & 16.59 & 14.66 & $0.20 \%$ \\
& 2 & 16.88 & 18.64 & 16.58 & $1.81 \%$ \\
$\mathrm{D}_{90}$ cup-down & 1 & 15.37 & 16.42 & 14.56 & $5.56 \%$ \\
& 2 & 16.82 & 18.51 & 16.45 & $2.25 \%$ \\
$\mathrm{D}_{90}$ cup-up & 1 & 15.37 & 16.82 & 14.88 & $3.29 \%$ \\
& 2 & 16.75 & 18.83 & 16.76 & $0.06 \%$ \\
\hline \hline
\end{tabular}


One of these effects is specific to the manufacturing technique that had been adopted, as during the course of this study it was realized that vacuum forming leads to considerable nonuniformity in the thickness of the manufactured shells. The remaining effects are of a general kind and would affect any future development of this kind of structures.

A general issue that has been identified is the difficulty of defining accurately the initial configuration for a flexible reflector structure. Because of the combination of a high compliance and self-weight effects in a structure of curved shape, it appears that the only practical way of determining such an initial reference configuration when the structure is tested in a gravity environment may be to compare the results of tests with different initial conditions to results from a numerical model of the structure.

\section{References}

[1] Anonymous, "Hughes Graphite Antennas Installed on MSAT-2 Craft," Space News, November 1994.

[2] Robinson, S. A., "Simplified Spacecraft Antenna Reflector for Stowage in Confined Envelopes," European Patent EP 0534110 A1, filed 31 March 1993, European Patent Application filed by Hughes Aircraft Company.

[3] Tan, L. T., and Pellegrino, S., "Thin-Shell Deployable Reflectors with Collapsible Stiffeners: Part 1, Approach," AIAA Journal, Vol. 44, No. 11, November 2006, pp. 2515-2523. doi: $10.2514 / 1.16320$

[4] "Polytec Scanning Vibrometer, Software Manual 7.1," Manual.
[5] PCB Piezotronics Vibration Division, Model 086D80 Impulse hammer, Installation and Operating Manual, PCB Piezotronics, Inc., Depew, NY, http://www.pcb.com/contentstore/docs/PCB_Corporate/Vibration/ products/Manuals/086E80.pdf [retrieved Nov. 2011].

[6] Ewins, D. J., Modal Testing: Theory and Practice, Wiley, New York, 1995.

[7] SIMULIA, ABAQUS/Standard Version 6.7, Providence, RI, 2007.

[8] Coxeter, H. S. M., Introduction to Geometry, Wiley, New York, 1980.

[9] Korn, G. A., and Korn, T. M., Mathematical Handbook for Scientists and Engineers, McGraw-Hill, New York, Ch. 2, p. 32.

[10] Fahy, F. J., Sound and Structural Vibration: Radiation, Transmission, and Response, Academic Press Ltd., New York, 1985.

[11] Southwell, R. V., "On the Free Transverse Vibration of a Uniform Circular Disc Clamped at its Centre; and on the Effects of Rotation," Proceedings of the Royal Society of London, Vol. 101, No. 709, 1922, pp. 133-153. doi:10.1098/rspa.1922.0032

[12] Lamb, H., "On Vibrations of an Elastic Plate in Contact with Water," Proceedings of the Royal Society of London, Series A: Mathematical and Physical Sciences, Vol. 98, No. 690, 1920, pp. 205-216. doi:10.1098/rspa.1920.0064

[13] Kukathasan, S., and Pellegrino, S., "Vibration of Prestressed Membrane Structures in Air," 43rd SDM Conference, AIAA Paper 2002-1368, April 2002.

[14] Achenbach, J. D., Wave Propagation in Elastic Solids, North-Holland Publishing Company, Amsterdam, 1976.
B. Epureanu Associate Editor 